\title{
Original
}

\section{Pathophysiology and Maturation of the Transverse Carpal Ligament in Idiopathic Carpal Tunnel Syndrome - Clinical, Pathological and Immunohistochemical Study -}

\author{
Hirotsune Hirahara, Katsunori INAGAKI, Etsuo FuJIMaKI \\ and Miki Kushima*
}

\begin{abstract}
The etiology of idiopathic carpal tunnel syndrome remains poorly understood. This study investigated the relationship between idiopathic carpal tunnel syndrome and transverse carpal tunnel ligament histology, especially clinical severity of this disease and immunohistochemistry of the ligament ${ }^{1)}$. Ligament biopsy specimens were obtained from eleven wrists during operation for carpal tunnel release, and one control specimen was obtained. After staining of different slices from each specimen with $\mathrm{HE}$ and antibodies to collagen types $\mathrm{I}$ to $\mathrm{VI}$, each slice was classified into 3 grades based on pathology, where grade 1 was the least and 3 the greatest pathological progression. Out of eight specimens from mild carpal tunnel syndrome patients, 4 were grade $\mathrm{H}-1$, and the other 4 were grade $\mathrm{H}-2$ based on $\mathrm{HE}$ staining. Out of 4 specimens from moderate to severe carpal tunnel syndrome patients, 1 was grade $\mathrm{H}-2$ and the other 3 were grade $\mathrm{H}-3$ with HE. Grades for anti-type I collagen were C-1 in 6 out of 8 mild CTS patients, whereas the grades were $\mathrm{C}-2$ or C-3 in 3 out of 4 moderate to severe patients. Grades for anti-type III collagen were $\mathrm{C}-1$ in 4 out of 4 mild patients whereas the grades were C-2 or C-3 in all 4 moderate to severe. The sum of type I and type III grades were 2 in control patients, $2-3$ in the mild CTS patients, 4 in the moderate patients and 6 in the severe patients.

There was a correlation between the severity of this disease and the degree of transverse ligament pathology stained with HE, anti-type I and III collagen antibodies. These suggest that disturbance of the maturation of collagen fibers in the transverse carpal ligament is an important factor in idiopathic carpal tunnel syndrome.
\end{abstract}

Key words : carpal tunnel syndrome, transverse carpal ligament, anti-collagen immuno-histochemistry, pathophysiology, maturation of collagen fiber

\section{Introduction}

Idiopathic carpal tunnel syndrome is the most common entrapment neuropathy of the median nerve at the transverse carpal ligament. This syndrome frequently occurs in women

Department of Orthopedic Surgery, Showa University School of Medicine, 1-5-8 Hatanodai, Shinagawa-ku, Tokyo 142-8555, Japan.

* Department of Hospital Pathology, Showa University School of Medicine. 
aged from 40-60 years. The transverse carpal ligament between the carpal bones is the floor of the carpal tunnel. This ligament is a thick fibrous band attached on the radial side to the tuberosity of the scaphoid and a portion of the trapezium and on the ulnar aspect to the pisiform and hook of the hamate. The median nerve lies superficial directly beneath this ligament. Although the disease is very common, in many patients little specific cause can be found and its pathophysiology is still not clearly understood. To date, many researchers have investigated the pathoanatomy of the flexor synovium or synovial sheath, and speculated that idiopathic carpal tunnel syndrome is caused by mechanical and chronic tenosynovitis and increase in volume of the tunnel. The purpose of this study was to investigate the histology of the transverse carpal ligament in patients with idiopathic carpal tunnel syndrome, and to analyze expression of collagen subtypes I-VI, and clarify the relationship between the degree of maturation of the collagen fibers and the severity of the median neuropathy. Our hypothesis was that disturbance of the maturation of collagen fibers in the transverse carpal ligament may be an important factor in idiopathic carpal tunnel syndrome.

\section{Materials and Methods}

\section{Materials}

Twelve transverse carpal ligaments were obtained from 11 patients diagnosed with idiopathic carpal tunnel syndrome despite amyloidosis who underwent open surgery at our department, and one ligament was obtained from an injured patient as a control. The patients were 1 male and 10 females with a mean age of 51.4 years, and 6 ligaments were from the right hand and 6 from the left. All patients favored the right hand. All females were housewives and one male was a desk worker. The diagnosis of idiopathic carpal tunnel syndrome was established by patient interview, physical examination and electrophysiological study (Table 1). The electrophysiological diagnosis criteria were a terminal latency

Table 1. Clinical symptoms and neural findings in each carpal tunnel syndrome patient

\begin{tabular}{|c|c|c|c|c|c|c|c|c|c|}
\hline Cases & Age & Sex & Diagnosis & $\begin{array}{l}\text { Op. } \\
\text { Side }\end{array}$ & $\begin{array}{c}\text { Chief } \\
\text { Complaint }\end{array}$ & $\begin{array}{c}\text { Phalen } \\
\text { Test }\end{array}$ & $\begin{array}{l}\text { Tinel } \\
\text { Sign }\end{array}$ & $\begin{array}{c}\mathbf{M M T}^{* *} \\
(\mathbf{A P B})\end{array}$ & $\begin{array}{l}\text { APB }^{* * *} \\
\text { Atrophy }\end{array}$ \\
\hline 1 & 56 & $\mathrm{~F}$ & Bil-CTS & Lt & Numbness & + & + & 2 & + \\
\hline 2 & 57 & $\mathbf{M}$ & Lt-CTS & $\mathbf{L t}$ & Numbness & $2+$ & $1+$ & 5 & - \\
\hline 3 & 27 & $\mathrm{~F}$ & Bil-CTS & $\mathrm{Lt}$ & Numbness & - & - & $5-$ & - \\
\hline 4 & 56 & $\mathbf{F}$ & Bil-CTS & Rt & Numbness, Night pain & - & $+1-$ & 4 & + \\
\hline 5 & 41 & $\mathbf{F}$ & Bil-CTS & Lt & Numbness, Night pain & - & + & 4 & + \\
\hline 6 & 78 & $\mathbf{F}$ & Rt-CTS & $\mathbf{R t}$ & Numbness & + & $2+$ & 3 & $2+$ \\
\hline 7 & 28 & $\mathbf{F}$ & Rt-CTS & $\mathbf{R t}$ & Numbness & + & + & 5 & - \\
\hline 8 & 46 & $\mathrm{~F}$ & Rt-CTS & Rt & Numbness & - & - & $5-$ & - \\
\hline $9^{*}$ & 56 & $\mathrm{~F}$ & Bil-CTS & $\mathbf{R t}$ & Numbness & + & + & 4 & + \\
\hline $10^{*}$ & 57 & $\mathrm{~F}$ & Bil-CTS & $\mathbf{L t}$ & Numbness & + & $+1-$ & 4 & $+1-$ \\
\hline 11 & 54 & $\mathrm{~F}$ & Rt-CTS & Rt & Numbness & + & + & $4-$ & $+1-$ \\
\hline 12 & 47 & $\mathbf{F}$ & Lt-CTS & Lt & Numbness & - & - & 4 & + \\
\hline 13 & 29 & $F$ & Control & $\mathbf{R t}$ & & & & & \\
\hline
\end{tabular}

Case $9,10^{*}$ : same patient

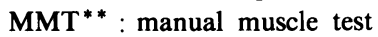

$\mathrm{APB}^{* * *}$ : abductor pollicis brevis 


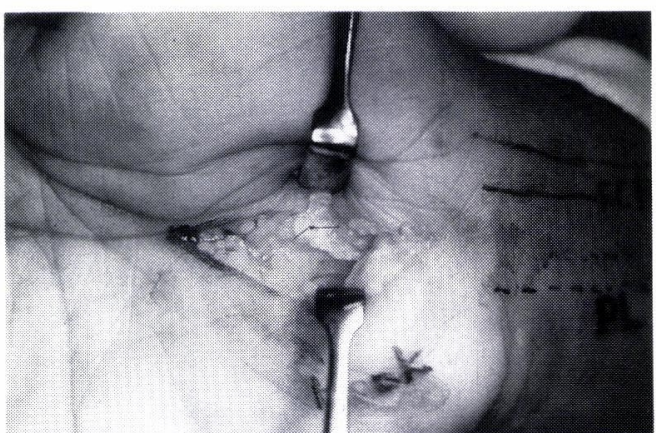

A

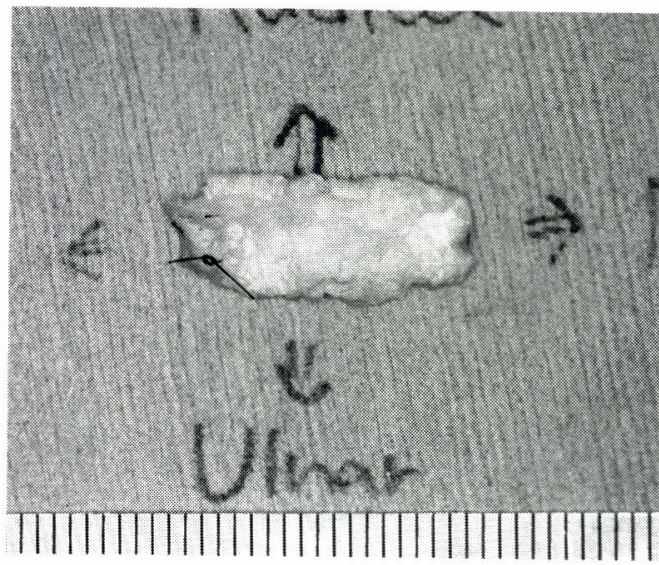

B

Fig. 1. Resection of a transverse carpal ligament with carpal tunnel syndrome

We dissected the ligament as far as possible on the ulnar side and the median nerve was decompressed. Also, before sectioning a 4-0 nylon suture was tied at the proximal part of the transverse carpal ligament on the palmar side.

(TL) of the abductor pollicis brevis (APB) muscle of more than $4.2 \mathrm{msec}$ and a sensory conduction velocity $(\mathrm{SCV})$ of less than $45 \mathrm{~m} / \mathrm{sec}$.

\section{Dissection of the Transverse Carpal Ligaments}

Open wrist surgery was performed for 12 hands diagnosed with idiopathic carpal tunnel syndrome, in which the patients requested surgical treatment. The standard method in which the skin incision does not exceed the wrist skin line, or a skip incision ${ }^{2}$, which is frequently performed at our department, was employed in the operations. After confirming the transverse carpal ligament by direct vision, we dissected the ligament as far as possible on the ulnar side and the median nerve was decompressed. Then, the ligament was sectioned and a specimen of about 5 to $6 \mathrm{~mm}$ was taken from the radial side. Also, before sectioning, a 4-0 nylon suture was tied at the proximal part of the transverse carpal ligament on the palmar side, which was subsequently used as a mark for sectioning and follow-up (Fig. 1). In the single control case, the median nerve was ruptured in an injury, and a specimen of the ligament was sectioned during the nerve suture. Before taking the tissue specimens, all patients were given a full explanation and informed consent was obtained.

\section{Methods}

The 13 transverse carpal ligaments extirpated during surgery were formalin-fixed, paraffin-embedded, and sections were made. HE staining, and the distribution of collagen types I to VI was investigated using the unlabelled antibody technique (LSAB-POD method) of the enzyme antibody technique. The relationship between the histological picture and the severity of the neurological severity of the manifestations was investigated. The neurological severity in the 12 hands with idiopathic carpal tunnel syndrome was comprehensively evaluated, mainly on the basis of the clinical findings and the 
Table 2. Neurological severity in all subjects

\begin{tabular}{|c|c|c|c|c|c|c|c|c|c|c|c|}
\hline \multirow{2}{*}{ Case } & \multirow{2}{*}{ Age } & \multirow{2}{*}{ Sex } & \multirow{2}{*}{ Diagnosis } & \multirow{2}{*}{$\begin{array}{l}\text { Op. } \\
\text { Side }\end{array}$} & \multicolumn{2}{|c|}{ T.L. ${ }^{*}$} & \multicolumn{2}{|c|}{$\mathrm{SCV}^{* *}$} & \multicolumn{2}{|c|}{ SNAP ${ }^{* * *}$} & \multirow{2}{*}{ Severity } \\
\hline & & & & & Rt. & Lt. & Rt. & Lt. & Rt. & Lt. & \\
\hline 2 & 57 & $\mathbf{M}$ & Lt-CTS & $\mathbf{L t}$ & 3.4 & 3.8 & 45.8 & 50.6 & 1 & 2 & Mild \\
\hline 3 & 27 & $\mathrm{~F}$ & Bil-CTS & $\mathrm{Lt}$ & 6.6 & 5.9 & 50.6 & 38.9 & 2 & 2 & Mild \\
\hline 4 & 56 & $\mathbf{F}$ & Bil-CTS & $\mathbf{R t}$ & 5.1 & 5.2 & 38.9 & 40.7 & 2 & 1 & Mild \\
\hline 5 & 41 & $\mathbf{F}$ & Bil-CTS & $\mathrm{Lt}$ & 6.7 & 5.9 & 38.1 & 34.6 & 1 & 2 & Mild \\
\hline 7 & 28 & $\mathbf{F}$ & Rt-CTS & $\mathbf{R t}$ & 4.2 & 3.5 & 45.4 & 54.6 & 1 & 1 & Mild \\
\hline 8 & 46 & $\mathbf{F}$ & Rt-CTS & $\mathbf{R t}$ & 5.6 & 3.1 & 32.8 & 50.3 & 2 & 1 & Mild \\
\hline 9 & 56 & $\mathbf{F}$ & Bil-CTS & $\mathbf{R t}$ & 10.6 & 10 & 26.5 & 17.7 & 2 & 3 & Mild \\
\hline 12 & 47 & $\mathbf{F}$ & Lt-CTS & Lt & 3 & 3.9 & 57.4 & 36.5 & 1 & 2 & Mild \\
\hline 10 & 57 & $\mathrm{~F}$ & Bil-CTS & $\mathrm{Lt}$ & 10.6 & 10 & 26.5 & 17.7 & 2 & 3 & Moderate \\
\hline 11 & 54 & $\mathbf{F}$ & Rt-CTS & $\mathbf{R t}$ & 8.9 & 4.5 & 20.2 & 40.5 & 3 & 2 & Moderate \\
\hline 1 & 56 & $\mathbf{F}$ & Bil-CTS & $\mathbf{L t}$ & 5.6 & 7.3 & 39.2 & NR & 2 & 3 & Severe \\
\hline 6 & 78 & $\mathbf{F}$ & Rt-CTS & $\mathbf{R t}$ & 9.9 & 4.8 & NR & 40.8 & 3 & 2 & Severe \\
\hline 13 & 29 & $\mathbf{F}$ & Control & $\mathbf{R t}$ & & & & & & & \\
\hline
\end{tabular}

electrophysiological assessment. The clinical assessment was made by a surgeon masked to the histological evaluation, and the microscopic assessment was made by a pathologist masked to the clinical evaluation.

1) Electrophysiological Evaluation and Severity of Neurological Manifestations

The electrophysiological evaluation was classified into three types based on analysis of the waveform of the sensory nerve action potential (SNAP) by Inagaki et al. ${ }^{3)}$; type 1, reduction in the amplitude or extension of the latency period; type 2, temporal dispersion; and type 3 , remarkable reduction of the amplitude and multiple phases.

The clinical findings are shown in Table 1, and the severity of the neurological manifestations was classified into three groups based on the clinical course; mild type, moderate type, and severe type. Mild carpal tunnel syndrome (CTS) includes SNAP Types 1 and 2 with or without muscular atrophy, moderate CTS includes SNAP Type 3 without muscular atrophy, and severe CTS includes SNAP Type 3 with muscular atrophy (Table 2). In the present study, cases with complete degeneration of the abductor pollicis brevis (APB) with muscle power of [0] or [1] by manual muscle testing (MMT) were excluded.

2) HE Staining and Classification

According to the conventional method, the specimens were stained with HE. The results of $\mathrm{HE}$ staining were classified into Grades $\mathrm{H}-1$ to $\mathbf{H}-3$. In grade $\mathrm{H}-1$, the fibers of the ligament were not disordered. In grade $\mathrm{H}-2$, the principal fascicules of the ligament were reduced with little new fiber formation. In grade $\mathrm{H}-3$, the principal fascicules of the ligament were reduced and loosened with abundant new fiber formation among them. All cases were negative for Congo red stain.

3) Anticollagen Staining ${ }^{4,5)}$ (Labeled Avidin Biotin Peroxidase) and Classification

The procedure for treatment of ligament and anticollagen staining is shown in Table 3 . Polyclonal antibody (Japan Tanner Corporation) was used as the primary antibody for 
Table 3. Technique of Collagen Staining

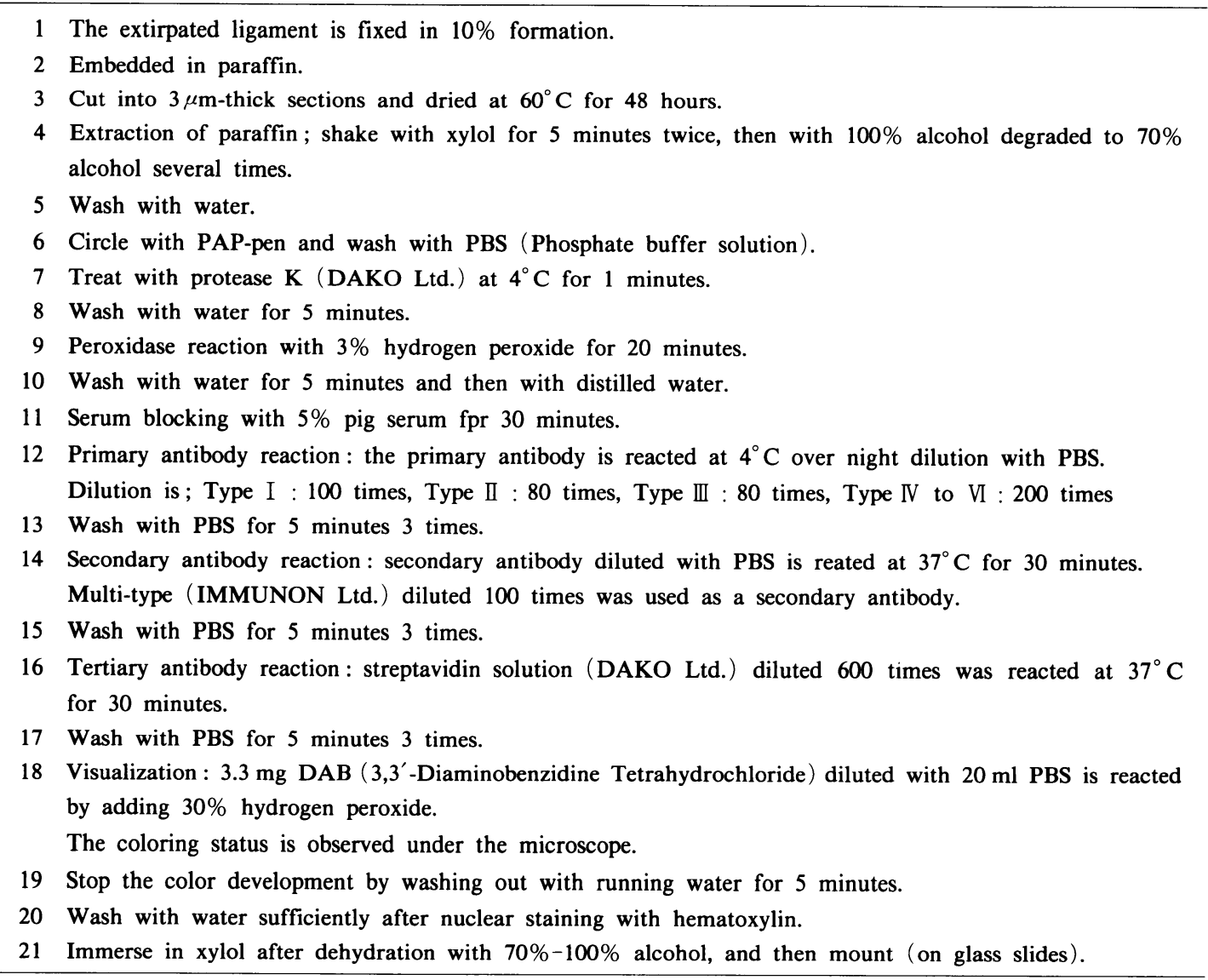

anticollagen staining. The results of staining for each collagen were classified into Grades $\mathrm{C}-1$ to C-3. In staining for collagen type, the principal fascicules of the ligaments were stained darkly in Grade C-1, less darkly stained in Grade C-2, and moderately stained in Grade C-3. In staining for collagen type II, the specimens were classified by the appearance of chondrocytes. No chondrocytes were seen in Grade C-1, were suspected in Grade C-2, and were manifest in Grade C-3. In staining for collagen type III, the classification was based on staining of newly-formed fibers. The newly-formed fibers were stained faintly in Grade C-1, stained moderately in Grade C-2, and stained intensély in Grade C-3. The staining for collagen types $\mathrm{V}, \mathrm{V}$ and VI was classified into Grades C-1 to $\mathrm{C}-3$, the same as collagen type III on the basis of staining for newly formed fibers.

4) Statistical analysis

We used Fishers method for statistical analysis.

\section{Results}

Relationship between electrophysiological severity and clinical stages

Eight hands were classified based on clinical findings as mild type $(66.7 \%)$, two hands 
Table 4. Staining grade for $\mathrm{HE}$ and antibodies to types I to VI collagen

\begin{tabular}{rcccccccc}
\hline \multirow{2}{*}{ Case } & \multirow{2}{*}{ Severity } & HE & I & II & III & I & V & VI \\
\hline 2 & Mild & H-1 & C-1 & C-1 & C-2 & C-1 & C-1 & C-1 \\
3 & Mild & H-1 & C-1 & C-1 & C-1 & C-2 & C-1 & C-1 \\
4 & Mild & H-1 & C-1 & C-1 & C-1 & C-3 & C-1 & C-2 \\
5 & Mild & H-2 & C-1 & C-1 & C-1 & C-2 & C-1 & C-2 \\
7 & Mild & H-2 & C-2 & C-1 & C-1 & C-1 & C-1 & C-2 \\
8 & Mild & H-2 & C-1 & C-1 & C-2 & C-1 & C-1 & C-3 \\
9 & Mild & H-2 & C-2 & C-1 & C-2 & C-1 & C-1 & C-3 \\
12 & Mild & H-1 & C-1 & C-1 & C-2 & C-1 & C-2 & C-2 \\
10 & Moderate & H-3 & C-2 & C-1 & C-2 & C-3 & C-2 & C-3 \\
11 & Moderate & H-2 & C-1 & C-1 & C-3 & C-3 & C-2 & C-3 \\
1 & Severe & H-3 & C-3 & C-2 & C-3 & C-3 & C-2 & C-3 \\
6 & Severe & H-3 & C-3 & C-2 & C-3 & C-3 & C-2 & C-3 \\
\hline 13 & Control & H-1 & C-1 & C-1 & C-1 & C-1 & C-1 & C-1 \\
\hline
\end{tabular}

were moderate type $(16.7 \%)$ and two were severe type $(16.7 \%)$. According to the electrophysiological classification for carpal tunnel syndrome, one was classified as Type 1 $(8.3 \%)$, seven were Type $2(58.3 \%)$ and four were Type $3(33.3 \%)$. As the electrophysiologic classification was determined in an early stage of carpal tunnel syndrome when symptoms are usually mild, there was no relationship.

\section{Relationship between pathology of the transverse carpal ligament and clinical severity}

Table 4 shows the relationship between the results of pathology of the transverse carpal ligament and clinical severity. Out of eight cases with mild CTS, there were 4 cases of grade H-1 (almost normal ligament without new fiber formation) and 4 cases of grade $\mathrm{H}-2$ (principal fascicules of the ligament were reduced with little new fiber formation). In two cases with moderate CTS, one was grade H-2 and the other was grade H-3 (marked reducedly and loosened fascicules of the ligament with abundant new fiber formation). Two severe CTS were H-3. There was a tendency for severe type carpal tunnel syndrome to have a higher grade of HE staining. In all 12 cases, there was no evidence of inflammatory cellular infiltration or vascularization.

In the collagen staining, we focused on collagen types I and III, and analyzed the relationship between the staining grade and clinical severity. For type I collagen staining, out of 8 patients with mild CTS, 6 cases were stained as grade C-1 $(75 \%)$, and the other 2 cases were stained as grade C-2 (25\%). In two cases with moderate CTS, one was grade C-1 and the other was grade C-2. In two cases with severe CTS, both were graded C-3 (Table 5).

For type III collagen staining, out of eight patients with mild CTS, four were grade C-1 (50\%), and four were grade C-2 (50\%). In two cases with moderate CTS, one was grade C-2 and another was grade C-3. Both cases with severe CTS were graded C-3 (Table 5).

Staining score and severity of median neuropathy

The sum of the grades for type I and type III collagen for each patient was defined as 
Table 5. Staining grade of HE, type I and III collagen in mild, moderate and severe carpal tunnel syndrome

\begin{tabular}{llccc}
\hline \multicolumn{1}{c}{ Severity } & & Mild & Moderate & Severe \\
\hline \multirow{2}{*}{ HE } & Grade H-1 & 4 & & \\
& Grade H-2 & 4 & 1 & \\
& Grade H-3 & & 1 & 2 \\
\multirow{2}{*}{ Collage type I } & Grade C-1 & 6 & 1 & 2 \\
& Grade C-2 & 2 & 1 & \\
\hline \multirow{3}{*}{ Collagen type III } & Grade C-3 & & & \\
& Grade C-1 & 4 & & 2 \\
\hline
\end{tabular}

Between severity and collagen type $I$, differences were considered significant at $\mathrm{p}<0.005$ (mild-severe), $\mathrm{p}<0.05$ (moderate-severe); mildmoderate was not significant.

Between severity and collagen type III , differences were considered significant at $\mathrm{p}<0.05$ (mild-moderate), $\mathrm{p}<0.01$ (mild-severe); moderatesevere was not significant.

Table 6. Relationship between staining score and neural severity in each specimen

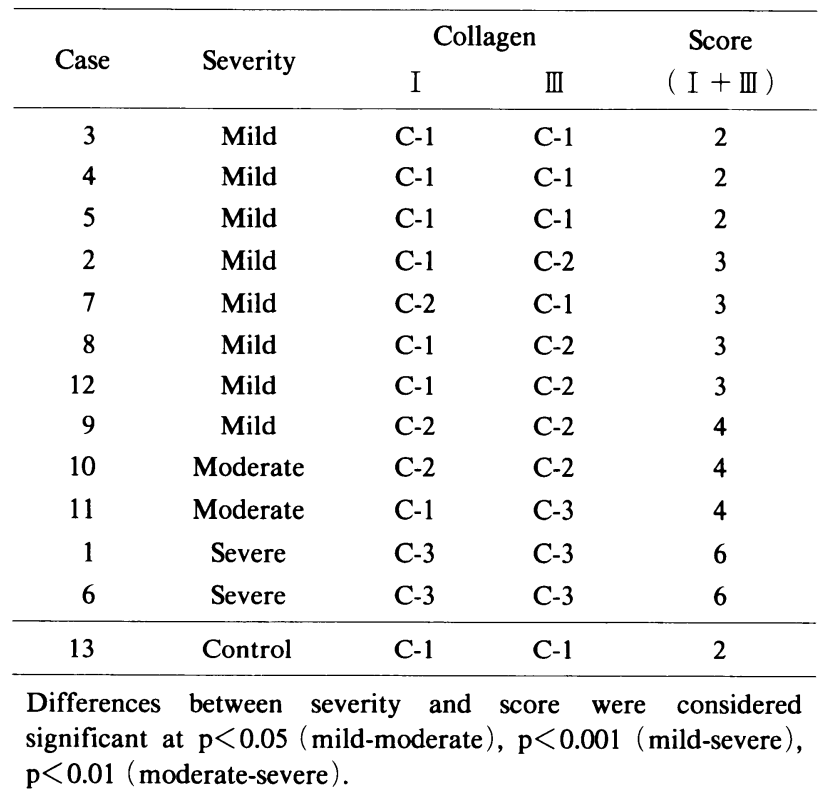

the 'staining score', and this was correlated with clinical severity. The average score was 2 in controls, 2.8 in mild stage, 4 in moderate and 6 in severe stage. There was a strong correlation between this point system and clinical severity (Table 6).

Other collagen subtypes

In type II collagen staining, all eight mild cases were grade C-1 (100\%). There were 
two moderate cases graded C-1 (100\%). Both severe-type specimens were stained grade C-2.

Type $\mathrm{V}$ collagen fibers had similar pathological characteristics to type III collagen; the regenerating fibers were well stained in the more severe type of carpal tunnel syndrome. We could not find any correlation between types IV and VI and severity of neuropathy.

\section{Discussion}

This study investigated the pathophysiology of the transverse carpal ligament using $\mathrm{HE}$ staining and specific antibodies to collagen types I to VI. Results suggested that as neural severity increased, there was derangement of the primary collagen fiber bundle and reduction in thick fiber bundles. The surrounding regenerating neofibers appeared as irregular constricted fibrous bands. Previous anti-collagen antibody immunohistochemistry studies using LSAB-POD reported that collagen type I was the mature collagen fiber, type II was fibrils of hyaline cartilage, type III was fine reticular fibers, type IV was vascular basement membrane, and type $\mathrm{V}$ was exocytoskeletal and pericellular connecting elements ${ }^{6-8)}$. Collagen type VI has not been investigated yet. Our results suggested that as the severity of carpal tunnel syndrome advanced, the fibrosis and degeneration of the collagen bundles and the formation of regenerating fibers in the surroundings increased. Idiopathic carpal tunnel syndrome therefore originates from thickening of the transverse carpal ligament with increased fibrosis. In the ligament maturation process, there may be some transformation of the fiber due to disturbance in the normal maturation process of collagen. In the first stage, the turn-over rate in maturation of the collagen fibers might be blocked, and in the second stage irreversible and fibrotic changes to the transverse carpal ligament could happen. The changes in collagen type III histology indicated the second process. After the disturbance of the maturation of the collagen fibers, the secondary changes of the fibers, detachment and irregularity of the bundles, and interposed newborn regenerating small fibers may appear in response to the degeneration (Fig. 2).

Taniguchi et al. ${ }^{9)}$ reported that chondrocyte-like cells which are positive for PAS staining analysis are commonly observed in the transverse carpal ligament of idiopathic carpal tunnel syndrome patients, and that all of the cells were positive for S-100 protein. In contrast, Kobayashi et al. ${ }^{10)}$ reported that all cases they tested were negative for PAS staining analysis, and that there were no significant findings in the ligaments. In the present study, type II collagen, which is a marker of chondrocytes, was possibly stained in chondrocytelike cells in the neurologically severe cases, but these results were not clear. However, chondrocyte-like cells positive for type II collagen were observed in the transverse carpal ligament of patients with carpal tunnel syndrome receiving dialysis ${ }^{11)}$. Therefore, chondrocytes may be observed as the ligaments further degenerate.

Congestion or ischemia of the median nerve has been reported as one possible etiology of this syndrome. In 1976, Sunderland ${ }^{12)}$ reported venous congestion from hypotonia as a precursor to anoxic capillary endothelial damage and edema in carpal tunnel syndrome. This concept might explain the correlation between edema and vascular sclerosis. We agree with this hypothesis, however, these are the findings of circulation in the median nerve, and may represent a secondary change after fibrosis and thickening of the ligament and compression of the nerve ${ }^{13)}$. The nerve compression caused by the thickening of the ligament should be the first pathological change in idiopathic carpal tunnel syndrome.

Phalen ${ }^{14)}$ proposed that tenosynoviocyte fibrosis and chronic inflammatory cell invasion 

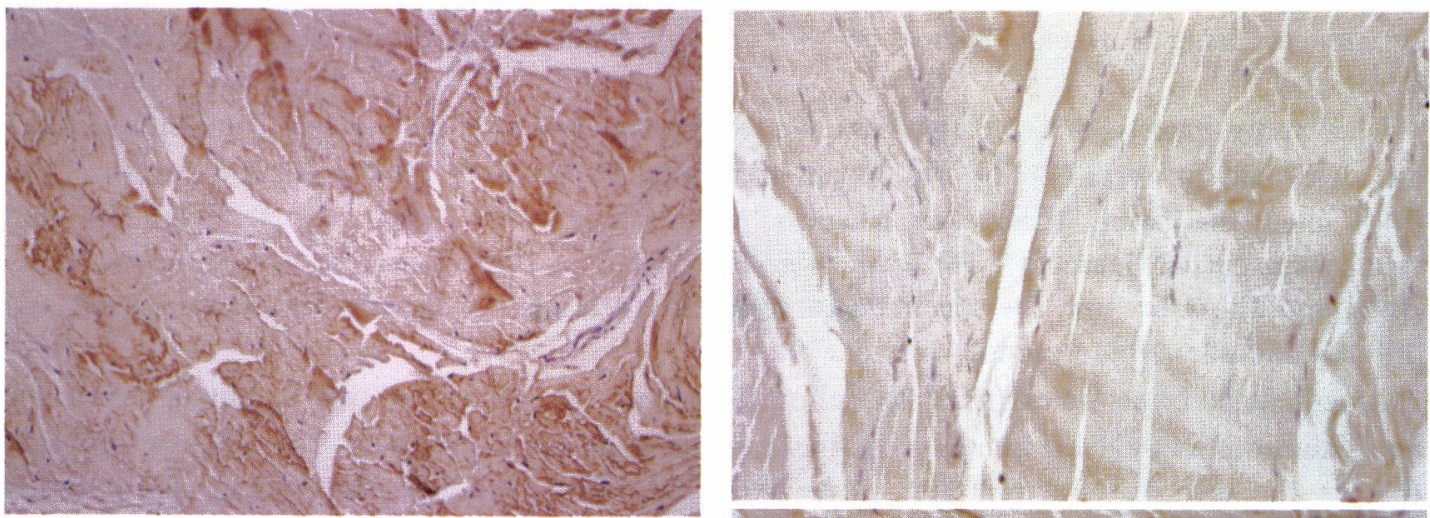

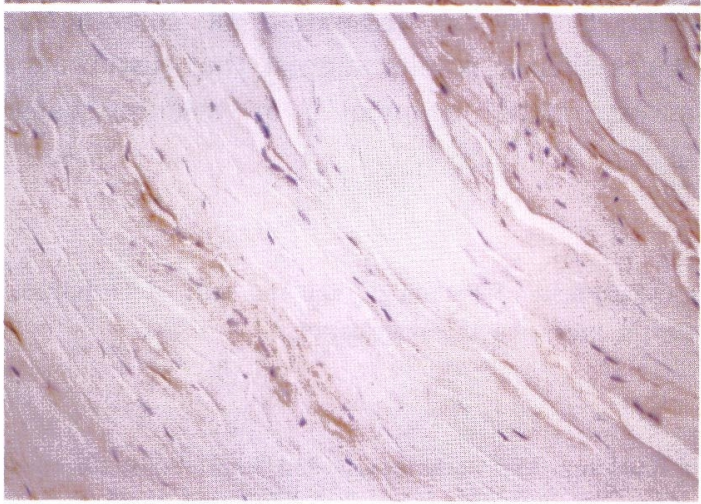

Fig. 2A Case 13, 29 y.o., Female, Control (upper) staining of Collagen types I $(\times 20)$ (lower) staining of Collagen types III $(\times 20)$

The fibers of the ligament were not disordered and were stained darkly for collagen I. There was no new fiber formation. Collagen III stain was grade C-1.

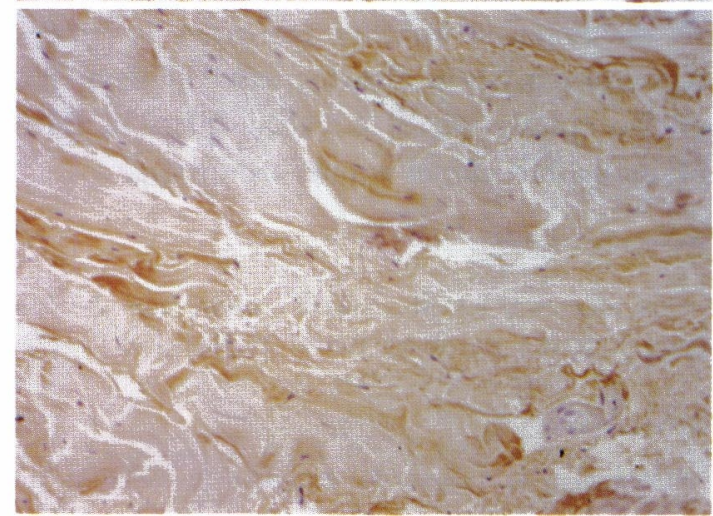

Fig. 2B Case 1, 56 y.o., Female, Severe type Idiopathic C.T.S.

(upper) staining of Collagen types I $(\times 20)$

(lower) staining of Collagen types III $(\times 20)$

The principal fascicules of the ligament were reduced and lost with abundant new fiber formation among them. The principal fascisules of the ligaments in grade C-3 were mildly stained, and new fibers were intensly stained in grade C-3.

are involved in the etiology of idiopathic carpal tunnel syndrome. In addition, Taniguchi et al. ${ }^{15)}$ reported that the presence of metaplastic chondrocytes plays a role in the hyperplasia and sclerosis of the transverse carpal ligament. Kobayashi et al. showed that hyperplasia of the synovial membrane surrounding the flexor digitorum profundus muscle (FDP) is responsible for idiopathic carpal tunnel syndrome.

There have been four major histological analyses of flexor tenosynovitis. Kerr et al. ${ }^{16)}$ reported that $96 \%$ of 625 cases showed chronic inflammation of the flexor tenosynovium. Fuchs et al. ${ }^{17)}$ noticed vascular sclerosis in $98 \%$ and edema in $85 \%$ of 177 cases. Scelsi et al. ${ }^{18)}$ identified collagen fibrosis in $80 \%$ of 50 cases. Schuind et al. ${ }^{19)}$ found no noteworthy abnormal findings other than hyperplasia. In the present study, no histological analysis of flexor tenosynovitis was performed, but the presence of synovitis as well as degeneration of the transverse carpal ligaments are considered to be responsible for the idiopathic carpal tunnel syndrome. Currently, endscopic open carpal tunnel release has proven a very effective treatment for idiopathic carpal tunnel. However, in the future, new chemical 
treatments using extracts from type $I$ and type III anticollagen are expected to be developed.

This study investigated the ligament named "transverse carpal ligament", but this name is not described in the PNA or the Japanese Society for Surgery of the Hand glossary. The PNA used the name "flexor retinaculum" to include both "volar carpal ligament" and "transverse carpal ligament". However, we described it as the "transverse carpal ligament" by BNA and JNA, because the specimen in this study was just "transverse carpal ligament" and did not include "volar carpal ligament"

In conclusion, we investigated the clinical severity of idiopathic carpal tunnel syndrome and examined collagen expression in the transverse carpal ligament. The results indicated that as the severity of carpal tunnel syndrome advanced, the irregularity and degeneration of the collagen bundles increased in type I collagen and the formation of surrounding regenerating fibers also increased in type III collagen. These results suggest that disturbance of the maturation of collagen fibers in the transverse carpal ligament is an essential cause of idiopathic carpal tunnel syndrome.

\section{Acknowledgments}

The authors thank Ms. Yoshiko Sasaki from the Second Department of Pathology, and the staff of Orthopedic Surgery, Showa University School of Medicine, for their help in preparing the mansucript.

\section{References}

1) Hirahara H, Inagaki K, Takigawa S, Fujimaki E and Kushima M: Pathological and immunohistochemical study of fibrous ligament in carpal tunnel syndrome. J Jpn Soc Surg Hand 14 : 1-3 (1997) in Japanese

2) Takigawa S, Fukushima K, Hirahara H, Inagaki $K$ and Fujimaki E: Open carpal tunnel release with "skip incision". J Jpn Soc Surg Hand 15 : 457-460 (1998) in Japanese

3) Inagaki K, Takigawa S, Hirahara H, Kawasaki K and Fujimaki E: Median nerve action potential in early stage carpal tunnel syndrome. J Jpn Soc Surg Hand $16:$ S127 (1999) in Japanese

4) Asano G : Immunohistochemical methods. Pathological technique for diagnosis and research. Medica globe Inc Tokyo (1992) in Japanese

5) Watanabe K and Nakane K : 3rd Edition Enzyme Antibody Method vol. 1, Gakusai kikaku (1992)

6) Ishida N, Onizuka $\mathrm{T}$ and Kushima $\mathbf{M}$ : Histopathologic Study of Bone Marrow Fibrous in Autopsy Cases-Normal Level and Increase in Myelodysplastic Syndrome-. J Showa Med Assoc 57: 198-208 (1997) in Japanese

7) Lisse I, Hasselbalch $\mathbf{H}$ and Junker P: Bone marrow stroma in idiopathic myelofibrosis and other haematological diseases, APMIS 99 : 171-178 (1991)

8) Gay S, Gay RE and Prohal JT: Immunohistological studies of bone marrow collagen, In Progress in Clinical and Biological Research. Vol. 154 Myelofibrosis and the biology of connective tissue, Berk PD (Ed), 291-306, Alan R. Liss, Inc., New York (1984)

9) Taniguchi Y, Tamaki T and Tanaka T: Histopathological studies on transverse carpal ligament in the carpal tunnel syndrome; appearance of metaplastic chondrocytes showed positive staining for S-100 protein. Seikeigeka 45 : 134-135 (1994) in Japanese

10) Kobayashi A, Futami T, Endou $T$ and Tsukamoto $Y$ : Idiopathic carpal tunnel syndrome: Operative and pathological studies of the flexor tendon synovium and transverse ligament. Kanto Tonrral of Orrhopedics and Tranmatology $30: 1-3$ (1999) in Japanese

11) Hirahara H, Takigawa S, Fukushima K, Tezuka M, Yoshizawa T and Fujimaki E: Clinical study of Carpal Tunnel syndrome in Hemodialysis patients. Kanto Journal of Orthopedics and Traumatology 29 : S42 (1998) in Japanese

12) Sunderland S: The nerve lesion in the carpal tunnel syndrome. J Neuro Neurosurg Psych 39: 615-26 (1976)

13) Johnston RB, Seiler JG, Miller EJ and Drvaric DM: The intrincic and extrinsic ligaments of the wrist. A correlation of collagen typing and histologic appearance. J Hand Surgery (British and European Volume), 20B (6) : 750-754 (1995)

14) Phalen GS: The carpal-tunnel syndrome. J Bone Joint Surg 48-A : 211-228 (1966) 
15) Taniguchi $Y$, Hayashi $M$, Hashizume $H$, Tamaki $T$ and Tanaka $T$ : Immunohistopathological studies on chondrocytoid cells transverse carpal ligament in the carpal tunnel syndrome. J Jpn Soc Surg Hand 12:360362 (1995) in Japanese

16) Kerr CD, Sybert DR and Albarracin NS : An analysis of the flexor synovium in ideopathic carpal tunnel syndrome: Report of 625 cases. J Hand Surg 17-A : 1028-1030 (1992)

17) Fuchs PC, Nathan PA and Myers LD : Synovial histology in carpal tunnel syndrome. J Hand Surg 16-A : 753-758 (1991)

18) Scelsi R, Zanlungo $M$ and Tenti $P$ : Carpal tunnel syndrome: anatomical and clinical correlations and morphological and ultrastructural aspects of the tenosynovial sheath. Ital J Orthop Traumatol 15: 75-80 (1989)

19) Schuind F, Ventura $M$ and Pasteels JL: Ideopathic carpal tunnel syndrome: histological study of flexor tendonsynovium. J Hand Surg 15-A : 497-503 (1990)

20) Ueba Y: Hand-function and anatomy. pp 178. KINPODO, Kyoto (1996) in Japanese

[Received December 3, 1999 : Accepted December 20, 1999] 